\title{
Correction to: A Semi-supervised Approach to Detect Malicious Nodes in OBS Network Dataset Using Gaussian Mixture Model
}

\author{
Md. Kamrul Hossain and Md. Mokammel Haque
}

\section{Correction to:}

Chapter "A Semi-supervised Approach to Detect Malicious

Nodes in OBS Network Dataset Using Gaussian Mixture

Model" in: G. Ranganathan et al. (eds.),

Inventive Communication and Computational Technologies,

Lecture Notes in Networks and Systems 89, https://doi.org/10.1007/978-981-15-0146-3_66

The original version of the book was published with incorrect corresponding author of Chapter "A Semi-supervised Approach to Detect Malicious Nodes in OBS Network Dataset Using Gaussian Mixture Model" "Muhammad Kamrul Hossain Patwary" (informal name) has been corrected to "Md. Kamrul Hossain" (official name). The chapter and book have been updated with the changes.

The updated version of this chapter can be found at https://doi.org/10.1007/978-981-15-0146-3_66 